\title{
Stability Analysis and Verification of self-balancing control system
}

\author{
Rong Qing ZHANG ${ }^{1}$ \& Lei ZHANG ${ }^{2}$ \& Shun Liang Sun \\ ${ }^{1}$ College of Mobile Telecommunications, Chongqing University of Posts and Telecommunications, \\ Chongqing, China \\ ${ }^{2}$ College of Mobile Telecommunications, Chongqing University of Posts and Telecommunications, \\ Chongqing, China \\ ${ }^{3}$ College of Mobile Telecommunications, Chongqing University of Posts and Telecommunications, \\ Chongqing, China
}

\begin{abstract}
Keywords: Index Terms -Self-balancing, stability Analysis, the centre of gravity equations of motion, error compares, PD controller.
\end{abstract}

\begin{abstract}
In view of the control method of present self-balancing system stability is so complex, and the controller requires high operation speed of the control system, so this paper presents a control method by using the centre of gravity equations of motion to analyze the system stability and maintain the system stability. This method derives the system center of gravity equation of motion by rigid body dynamics, and uses the control theory to analyze the system stability. In the case of instability, it applies the method of Kalman filter and error compares to eliminate the integral error of gyroscope and to acquire an accurate angle, at the same time ,using the angle, the angular velocity and the resultant acceleration which made by the corresponding parameters to make up the feedback branch configuration system poles, what's more, configuring the angle and angular velocity with appropriate parameters to make up the PD controller, at last completing the control of the system stability. Tests show that this method can reduce the complexity of the system operator and maintain the system stability.
\end{abstract}

\section{Introduction}

The main task of self-balancing system is getting the accurate angle and generating suitable control quantity in real time to control the system keeping at equilibrium state [1]. Due to the advantages of self balancing control system itself, it was widely used in the verticality control of the rocket, pendulum control, walking bipedal robot and two rounds of electric car vertical control, Therefore, the study of its stability is of great significance.

For the self-balancing system, getting accurate angle and taking some certain strategy to keep the system stability are the two important factors. Usually, theoretical analysis and stability Analysis of the self-balancing system is more complex, on the system Angle for general use kalman filter, complementary filter algorithm, etc, but the error still exists. On subsequent system balance control, general use fuzzy PID [2], LQR, or methods such as adaptive controller, and so on. In order to improve control accuracy, part of the algorithm should be subdivided control range [3], but it will make control rules increase by geometric, the so-called rule explosion problem, it will affect the real-time control.

In this paper presents a control method by using the centre of gravity equations of motion to analyze the system stability and maintain the system stability. This method derives the system center of gravity equation of motion by rigid body dynamics, and uses the control theory to analyze the system stability. In the case of instability, it applies the method of Kalman filter and error compares to eliminate the integral error of gyroscope and to acquire an accurate angle [4], at the same time, using the angle, the angular velocity and the resultant acceleration which made by the corresponding parameters to make up the feedback branch configuration system poles, what's more, configuring the angle and angular velocity with appropriate parameters to make up the PD controller, at last completing the control of the system stability. Tests show that this method can reduce the complexity of the system operator and maintain the system stability. 


\section{The establishment of the mathematical model}

According to the definition and applications of the self-balancing control system, its behavior characteristics are similar to inverted pendulum, so based on the analysis of the features of the selfbalancing control system, we can refer to the stability analysis of inverted pendulum [5]. But compared to the inverted pendulum, has its own characteristics the self-balancing control system, due to the adjust balancing mechanism of the mass of the self balancing control system relative to the mass of the whole system is very small, we can ignore the mass of the driving mechanism in the process of analysis, so it is not only simplify the theoretical analysis, but also simplify the design of the algorithm, reduce the complexity of the operation control system. The following will compare the inverted pendulum modeling and derive the self-balancing control system modeling.

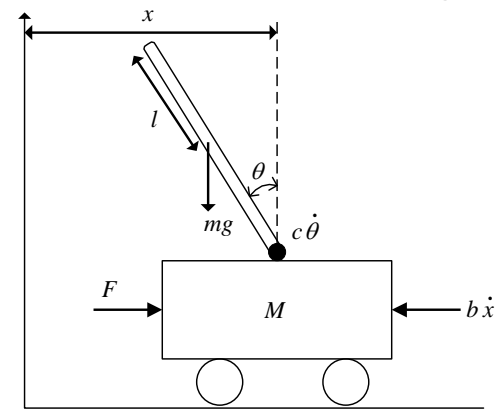

Fig. 1 Stress analysis for Inverted Pendulum.

Inverted pendulum system is shown in figure 1, stressing analysis to the horizontal and vertical direction and combining with the rigid body dynamics equation [6], the motion equation of inverted pendulum can be obtained:

$$
\begin{array}{r}
\left(J+m l^{2}\right) \ddot{\theta}+(m l \cdot \cos \theta) \ddot{x}=-c \cdot \dot{\theta}+m l \cdot g \cdot \sin \theta \\
(M+m) \ddot{x}+(m l \cdot \cos \theta) \ddot{\theta}=-b \dot{x}+(m l \cdot \sin \theta) \cdot \dot{\theta}^{2}+F
\end{array}
$$

In the expression, $x$ is the relative displacement of the benchmark for the car; $\theta$ is angular displacement of cycloid relative vertical; $J$ is inertia of the pendulum revolves around its center; $m$ is the quality of the pendulum; $M$ is the quality of the car; $l$ is The length of the pendulum of center of gravity to the rotation; $H$ is the car counter-acting force relative to pendulum; $V$ is the car counter-acting force relative to single pendulum; $c$ is the coefficient of friction of pendulum rotates; $b$ is the sliding coefficient of car, $F$ is external force acting on the car.

On the traditional analysis method of the self-balancing control system, the analysis method of the complex, operation for a long time to solve, therefore, simplified the system and reduce the complexity of the analysis are the two key solutions. Ignoring the mass of the driving mechanism, the simplified model of the inverted pendulum, that is the equivalent model of the self-balancing control system:

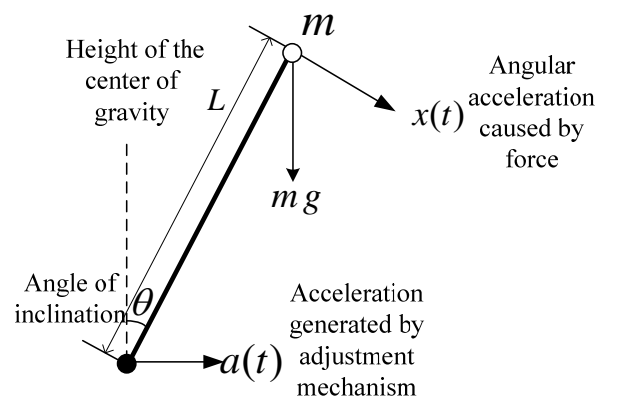

Fig.2 Equivalent chart for Self-balancing control system

Force analysis of the model as shown in figure 3: 


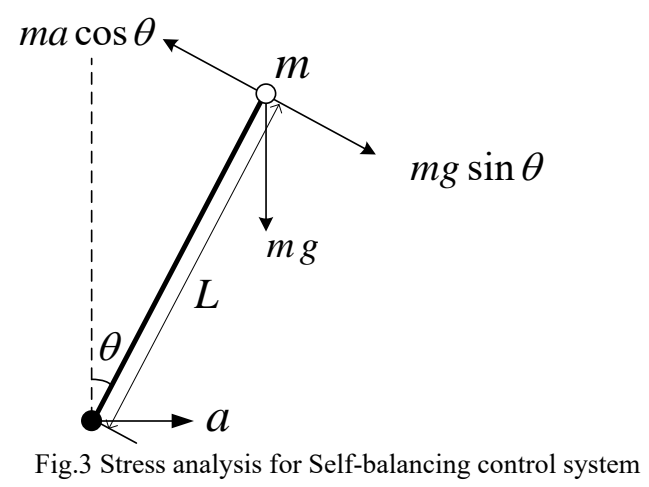

The model equivalent to inverted pendulum that can turn around the bottom of the pivot, its center of gravity from the bottom of the distance is $L$, the mass is $m$, assume that because of the external force disturbance cause the system leaning to the right and producing angular acceleration $x(t)$, an angle $\theta$ deviation from the vertical direction, ignoring the quality of the driving mechanism, after control system detects the angle, it makes drive mechanism drive the bottom of the fulcrum movement to the right, location in the center of mass is analyzed at the moment, It will be an additional inertial force, the force focuses on the system center of mass, which are opposite of direction of the drive acceleration, its value is $m a \cos \theta$, and it is proportional to the $a$, in the movement speed direction of center of mass:

$$
m L \frac{d^{2} \theta(t)}{d t^{2}}=m g \sin [\theta(t)]-m a(t) \cos [\theta(t)]+m L x(t)
$$

On both sides of the equation, eliminating $m$ :

$$
L \frac{d^{2} \theta(t)}{d t^{2}}=g \sin [\theta(t)]-a(t) \cos [\theta(t)]+L x(t)
$$

Usually, adjust speed is quickly in the actual process of adjustment, only small Angle deviate from the equilibrium position, so the equation of motion is simplified to:

$$
L \frac{d^{2} \theta(t)}{d t^{2}}=g \theta(t)-a(t)+L x(t)
$$

\section{The stability analysis and pole assignment}

\subsection{Stability analysis of the actual model}

The self-balancing control system is a typical single input and multiple outputs, nonlinear, high order, unstable system, when the object deviate from the equilibrium position, the direction of the restoring force and the displacement $s$ of object are obtuse angles $\alpha$ in an actual simple pendulum model, so tangential component force of gravity does negative work to swing object when pendulum deviate from the equilibrium position, meanwhile, because of air resistance and other factors, swinging objects stop at equilibrium position. But for the self-balancing control system, the center of mass is located at the top of supporting point, the system is in unstable equilibrium state, because of the existence of manufacturing error and other interference factors, it is very difficult to stop at the top of the supporting point for the self-balancing control system, once the center of mass deviate from the top of supporting point, the direction of the restoring force and the displacement $S$ of object are acute angle $\beta$, component force of gravity does positive work to swing object when self-balancing system deviate from the equilibrium position, the deviation degree of swing object is increased. Two states, respectively, as shown in figure 4 and figure 5. 

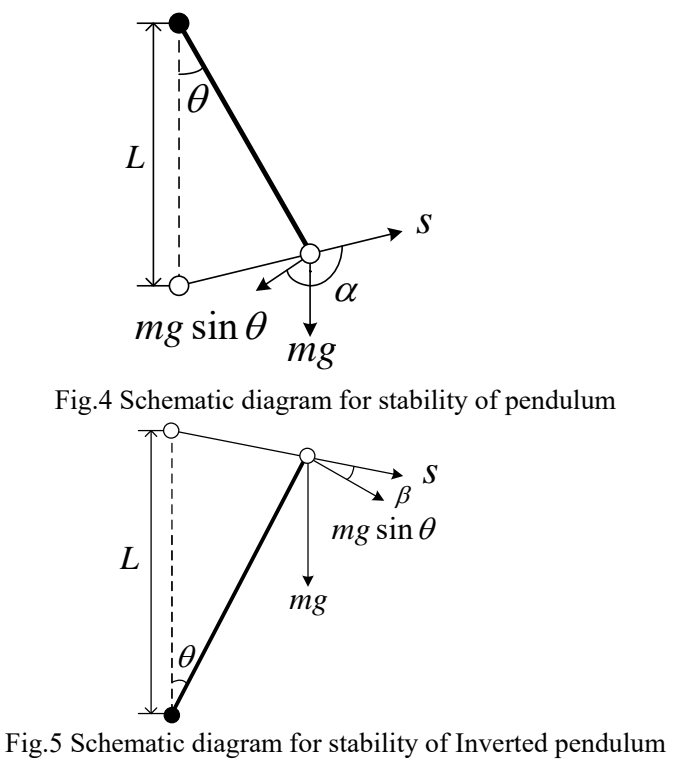

\subsection{Pole assignment of system}

By the formula 5, when the system is stationary, $a(t)=0$, so formula 5 into

$$
L \frac{d^{2} \theta(t)}{d t^{2}}=g \theta(t)+L x(t)
$$

For the system transfer function:

$$
H(s)=\frac{\Theta(s)}{X(s)}=\frac{1}{s^{2}-g / L}
$$

The system transfer function has two poles $s_{p}= \pm \sqrt{g / L}$, one of the poles is located in the S plane of the right half plane [7], So the system is unstable.

Drive mechanism drives the bottom of the fulcrum movement to the right or left to maintain system upright state, additional inertial force will be attached to the center of mass when drive mechanism makes adjustment, the force is opposite to the direction of the acceleration which form the movement of driving mechanism, the value is proportional to the acceleration of driving mechanism, as shown in figure 3 , restoring force addition to the center of mass is:

$$
F=m g \sin \theta-m a \cos \theta
$$

In the actual adjustment process, the adjust speed is very quick; the angle deviate from the equilibrium position is small, after linearizing the motion equation:

$$
F=m g \sin \theta-m a \cos \theta \approx m g \theta-m k_{1} \theta
$$

By the formula 8 , if $k_{1}>g$, the direction of the restoring force is opposite to the displacement, the restoring force will be addition to the center of mass when the object deviate from the equilibrium position, making the center of mass tends to move toward the equilibrium position.

In order to make the system stable in the equilibrium position as soon as possible, it is necessary to increase the damping force. The increasing number of damping force is proportional to the speed of the angle, but the direction is opposite, therefore, formula (8) into:

$$
F=m g \theta-m k_{1} \theta-m k_{2} \theta^{\prime}
$$

After increasing damping item, the self-balancing control system model equivalent to pendulum model, it can be stabled near the equilibrium position, so acceleration control algorithm of control system is:

$$
a_{k}=k_{1} \theta+k_{2} \theta^{\prime}
$$

$\theta$ is angle deviating from the equilibrium position, $\theta^{\prime}$ is angular velocity; $k_{1}$ and $k_{2}$ are proportionality coefficient. Two items are added as controlling quantity to regulate balance. Just be sure to $k_{1}>g$ and $k_{2}>0$, the self-balancing control system can keep upright state. $k_{2}$ determines damping coefficient, selecting appropriate damping coefficient can guarantee the system stability in 
the equilibrium position as soon as possible; $k_{1}$ determines whether the system is stable to vertical equilibrium position, it must be greater than the gravitational acceleration.

Through the analysis of the above, the diagram introduction of proportional and differential feedback control in control system is shown in figure 6:

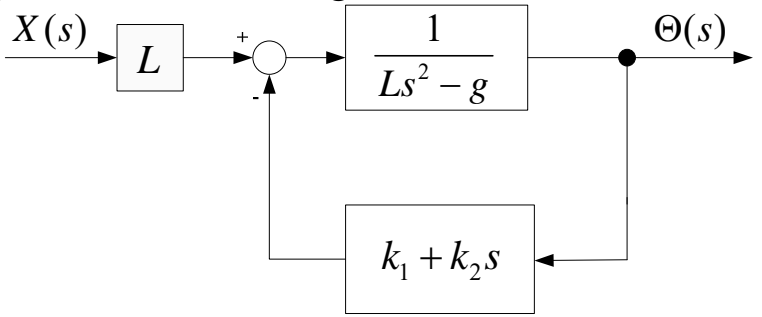

Fig.6 System block diagram for the introduction of proportional and differential

Angle feedback control is introduced in figure 6, control quantity of Proportional to the angle is the proportional control, controlling quantity of proportional to the angular velocity is the differential control, The differential parameters is equal to damping force, it can effectively suppress the oscillation of the system. After introduction of proportional and differential feedback control, system stability analysis is as follows:

The transfer function of system is:

$$
H(s)=\frac{\Theta(s)}{X(s)}=\frac{1}{s^{2}+\frac{k_{2}}{L} s+\frac{k_{1}-g}{L}}
$$

Now, the poles of system are:

$$
S_{p}=\frac{-k_{2} \pm \sqrt{k_{2}^{2}-4 L\left(k_{1}-g\right)}}{2 L}
$$

If the system is to remain stable, the two poles must be located the left half plane in S plane, to meet this condition, it is necessary to keep $k_{1}>g$ and $k_{2}>0$, So the self-balancing control system can keep equilibrium state. Adjusting appropriately proportionality coefficient and differential coefficient which can make the system quickly back to the equilibrium position and maintain stable state.

\section{Control strategy and the analysis of the results}

\subsection{Angle information acquisition and Processing}

Usually using acceleration and gyro sensors acquire angle information [8], because using a certain sensor separately can't acquire accurate angle information, combined with their advantages and disadvantages of the two kinds of sensors, we can get accurate angle. In order to get the angle information precisely, applying Kalman filter filters out interfering signals which from sensor and get useful angle [9]. In the process of application of kalman filter algorithm, the first step is integrating of the angular velocity which from gyro, calculate the angle from the gyro Angle_gyro:

$$
\text { Angle_gyro }=\text { Angle }+(\text { Gyro_data }-b) \cdot d t
$$

Gyro_data is angular velocity of gyro, comparing with angle from acceleration sensor get the current angle error Angle_error :

$$
\text { Angle_error }=\text { Angle_data }- \text { Angle_gyro }
$$

Angle_data is angle information from acceleration sensor which is calculated by MCU, according to the third formula of kalman filter:

$$
\text { Angle }=\text { Angle }+\mathrm{Kg} \cdot \text { Angle_error }
$$

Angle is on the left side of the equation is currently accurate angle after operation; Angle on the left side of the equation is accurate angle after kalman filter calculation previously. In the process of the kalman filter, through the recursive, kalman gain and covariance $\mathrm{P}$ must be calculated constantly, so that next angle and other parameters can be calculate, like $b$,so far, accurate angle 
information and gyroscope information has been calculated [10], the specific control process as shown in figure 7 :

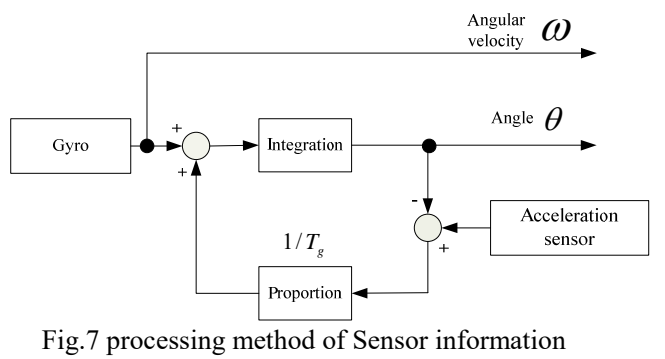

Comparing the angle from acceleration sensor with the angle from the gyro, the angle error can be calculated, through proportion amplifier, putting the error superposition to angular velocity, so that the integral error of gyroscope will be cleared. Then integrating angular velocity signal, it is very convenient to obtain relatively accurate angle.

\subsection{Producing control quantity}

Through differential operation of angle we can obtain angular velocity on mathematics, if angle is a part of the proportional, angular velocity is a part of the differential item, cooperating with the proper coefficient can compose the PD controller, so producing proper control quantity can guide drive mechanism to maintain equilibrium state, the specific control process as shown in figure 8:

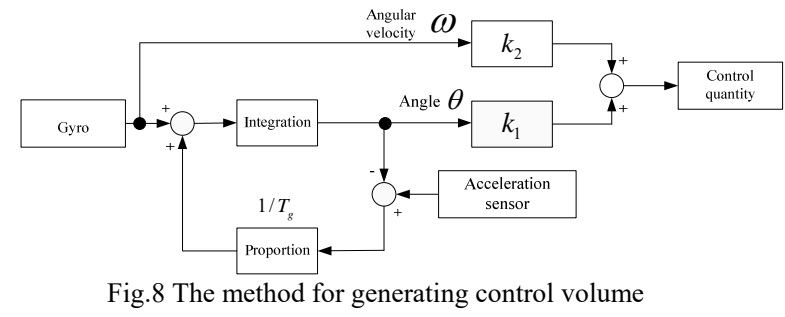

$k_{1}$ and $k_{2}$ are respectively Proportional coefficient and differential coefficient in figure 8 , the expression to produce control quantity as follow:

$$
\text { Control_quantity }=\mathrm{Kp} \cdot \text { Angle }+\mathrm{Kd} \cdot \mathrm{Gyro} \_ \text {real }
$$

$K p$ and $K d$ correspond to $k_{1}$ and $k_{2}$, appropriate adjustment and control them can get proper control quantity and complete balance adjustment.

\section{3 testing result and the analysis}

Key parameters will be sent to the computer when the system is running, because many important parameters can indicate whether system is working correctly and kalman filter can work correctly and how the effect of filter, as shown in figure 9. When the system is in equilibrium state, the angle is very small, in order to know the angle tracking effect, the angle data has been magnified 1500 times before transmitted to the computer, similar to figure 10 and figure 11 .

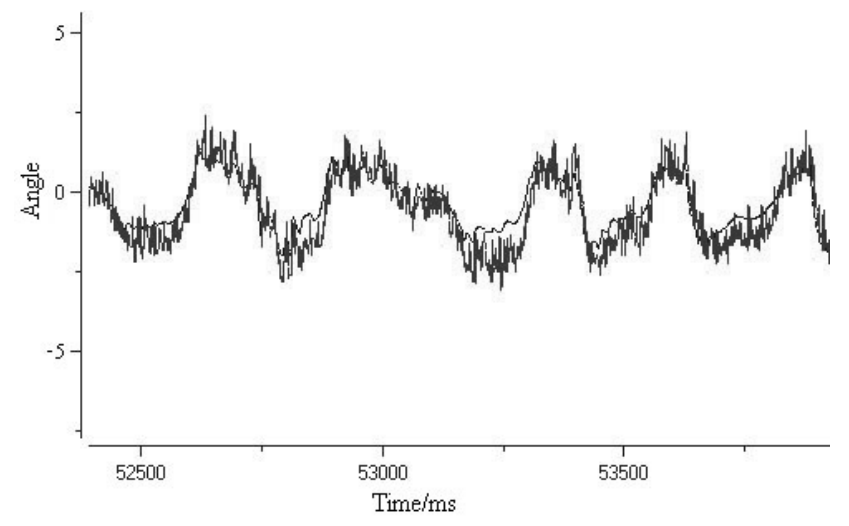

Fig.9 Angle Contrast before and after filtering 
The curve superposition of the high frequency signal is angle before filtering in figure 9, the smooth curve is angle after filtering, Figure 9 shows that the angle data becomes smooth after kalman filter, many of the jamming signal is cleared.

The production of control quantity need angle and angular velocity data, so accurate gyroscope data must be acquired, as shown in figure 10, the middle of the waveform is angle before and after filter, the larger waveform amplitude is angular velocity data.

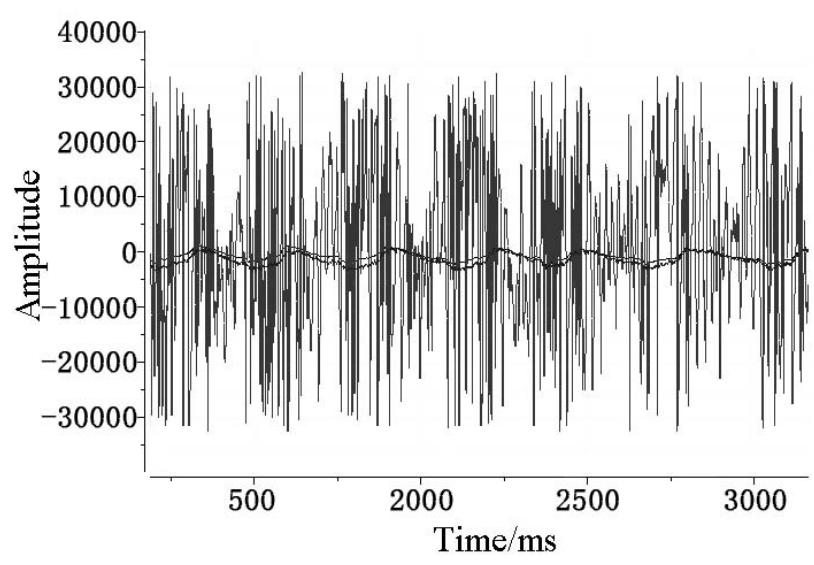

Fig.10 Angle and angular velocity before and after filtering

The curve in the middle of figure 11 is angle data, and the amplitude is smallest. The curve of control quantity is in the middle position, the amplitude value greater than the angle data and less than the angular velocity data. The largest amplitude value is angular velocity data. Because of angle data in the production of control quantity occupies a large proportion, the value of $K p$ is larger than the value $K d$ in the actual control.

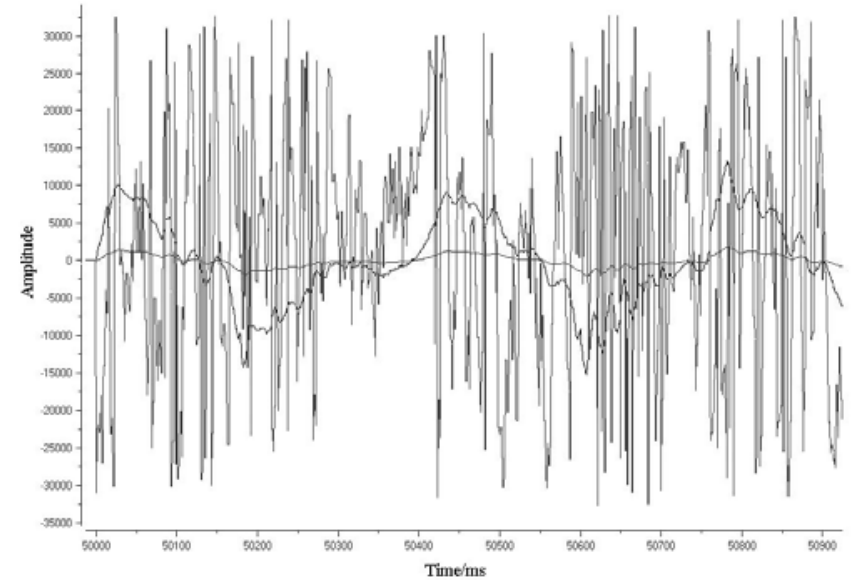

Fig.11 Waveform of angle, angular velocity after filtering and control quantity

\section{Conclusion}

This paper analyses the stability of the self-balance control system from theory to practice and deduces methods that get system to keep balance according to the theory and the actual analysis results. In order to prove the validity of the algorithm, test platform of the self-balance control system is designed, the system can calculate angle correctly through acceleration sensor, meanwhile, the validity of the improved algorithm is verified perfectly, the self-balance control system can work excellently.

\section{References}

[1] Jianguo Tao, Zongquan Deng. "Characteristic Analysis of A Two-Wheeled robotic Rover with Anti-Overturn Ability and Combinability," Proceedings of the 2006 IEEE/RSJ International Conference on Intelligent Robots and Systems.2006, Beijing, China 
[2] Sheng-Li QU, Xin-zhi ZHU. "Structures and Algorithms of a new dynamic fuzzy controller." Proceedings of the First Asia International Symposium on Mechatronics, 2004.

[3] Shiroma N, Matsumoto O, Kajita S. "Cooperative behavior of a wheeled inverted pendulum for object transportation." Proceedings of the 1996 IEEE/RSJ International Conference on Intelligent Robots and Systems. Osaka, 1996, pp.396-401.

[4] Q. J. Zhou. “Overview of Intelligent Control.” Information and Control, vol. 2, no.1, pp.38-45, March 1987

[5] Yanyan He, Chengzhi Shen. "Triple inverted pendulum system and analysis of its controllability." Journal of Beijing University of Aeronautics and Astronautics. 1996, pp.545-549.

[6] F.Grasser,A.D.Arrigo,S.Colombi. “a mobile Inverted Pendulum.” IEEE Trans. Industrial Electronics, 2002, pp.107-115.

[7] Dorf, Richard\&Robet H. Bishop. "Modern Control Systems.” Prentice-Hall,Unitd States of America.2001

[8] Hyungjik Lee, Seul Jung. "Balancing and navigation control of a mobile inverted pendulum robot using sensor fusion of low cost sensors." Mechatronics, 2012, pp.95-105.

[9] S. S. Young. "Attitude estimation using low cost accelerometer and gyroscope." Proceedings of the 7th korea-Russia International Sysposium on Science and Technology. 2003, pp.423-427.

[10] Y. Akcayir, Y. "Gyroscope drift estimation analysis in land navigation systems." Proceedings of the 2003 IEEE International Conference on Control Applications. 2003, pp.14881491. 